\title{
Recommendations on presenting LHC searches for missing transverse energy signals using simplified $s$-channel models of dark matter
}

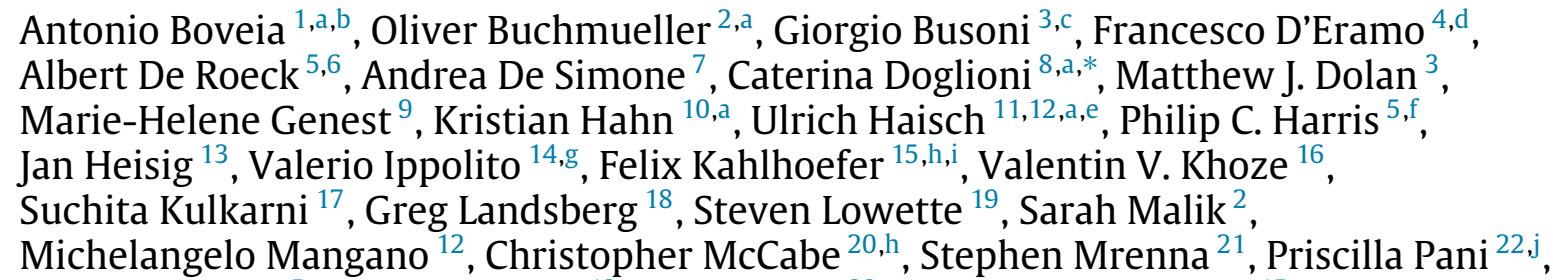
Tristan du Pree ${ }^{5}$, Antonio Riotto ${ }^{12}$, David Salek ${ }^{23}$, Kai Schmidt-Hoberg ${ }^{15}$, William Shepherd ${ }^{24, k}$, Tim M.P. Tait ${ }^{25, a}$, Lian-Tao Wang ${ }^{26}$, Steven Worm ${ }^{27,1}$, Kathryn Zurek ${ }^{28}$

${ }^{1}$ Ohio State University, USA

${ }^{2}$ High Energy Physics Group, Blackett Laboratory, Imperial College, Prince Consort Road, United Kingdom

${ }^{3}$ ARC Centre of Excellence for Particle Physics at the Terascale, School of Physics, University of Melbourne, Australia

${ }^{4}$ UC, Santa Cruz and UC, Santa Cruz, Inst. Part. Phys., USA

${ }^{5}$ CERN, EP Department, Switzerland

${ }^{6}$ Antwerp University, Belgium

${ }^{7}$ SISSA and INFN Sezione di Trieste, Italy

${ }^{8}$ Fysiska institutionen, Lunds universitet, Sweden

${ }^{9}$ LPSC, Universite Grenoble-Alpes, CNRS/IN2P3, France

${ }^{10}$ Department of Physics and Astronomy, Northwestern University, USA

${ }^{11}$ Rudolf Peierls Centre for Theoretical Physics, University of Oxford, United Kingdom

${ }^{12}$ CERN, TH Department, Switzerland

${ }^{13}$ Institute for Theoretical Particle Physics and Cosmology, RWTH Aachen University, Germany

${ }^{14}$ Laboratory for Particle Physics and Cosmology, Harvard University, USA

${ }^{15}$ DESY, Germany

${ }^{16}$ Institute of Particle Physics Phenomenology, Durham University, United Kingdom

${ }^{17}$ Institut für Hochenergiephysik, Österreichische Akademie der Wissenschaften, Austria

${ }^{18}$ Physics Department, Brown University, Providence, USA

${ }^{19}$ Vrije Universiteit Brussel IIHE, Belgium

${ }^{20}$ GRAPPA Centre of Excellence, University of Amsterdam, Netherlands

${ }^{21}$ FNAL, USA

22 Stockholm University, Sweden

${ }^{23}$ Nikhef, Netherlands

${ }^{24}$ Niels Bohr International Academy, Niels Bohr Institute, University of Copenhagen, Denmark

${ }^{25}$ Department of Physics and Astronomy, University of California Irvine, USA

${ }^{26}$ Enrico Fermi Institute and Department of Physics and Kavli Institute for Cosmological Physics, University of Chicago, USA

${ }^{27}$ Particle Physics Department, Rutherford Appleton Laboratory, United Kingdom

${ }^{28}$ University of California and LBNL, Berkeley, USA

\footnotetext{
* Corresponding author.

E-mail address: caterina.doglioni@hep.lu.se (C. Doglioni).

a Editor, Dark Matter Working Group organiser.

b Previously at CERN.

c Now at Max-Planck-Institut fur Kernphysik Heidelberg, Germany.

d Now Universita' di Padova, Italy.

e Now at Max Planck Inst. Munich.

f Now MIT, USA.

g Now INFN Rome.

$\mathrm{h}$ Editor.

i Now RTWH Aachen.

j Now DESY Zeuthen, Germany.

k Now University of Mainz, Germany.

1 Now University of Birmingham, United Kingdom.
} 


\section{A R T I C L E I N F O}

\section{Article history:}

Received 18 June 2019

Accepted 1 August 2019

\section{A B S T R A C T}

This document summarises the proposal of the LHC Dark Matter Working Group on how to present LHC results on $s$-channel simplified dark matter models and to compare them to direct (indirect) detection experiments.

(C) 2019 The Authors. Published by Elsevier B.V. This is an open access article under the CC BY license

(http://creativecommons.org/licenses/by/4.0/).

\section{Introduction}

The interpretation of searches for Dark Matter (DM) (or any other LHC physics result) requires that one assumes a model leading to the signal under consideration. This is necessary to compare searches across channels, searches at other centre-ofmass energies or at other collider experiments. The ATLAS and CMS experiments at the LHC coordinated in 2015 a joint forum to address this issue, in collaboration with theorists. This ATLAS/CMS DM Forum produced a report [1], providing a first set of concrete simplified DM models used by ATLAS and CMS to interpret their searches for missing transverse energy (MET) signatures.

At the end of the DM forum's activities, a formal LHC Dark Matter WG (LHCDMWG) was created, to continue the discussion and harmonisation of the way in which the LHC DM results are interpreted, reported and compared to those of other experimental approaches.

This document provides the LHCDMWG recommendations on how to present the LHC search results involving the $s$-channel models considered in [1] and how to compare these results to those of direct (DD) and indirect detection (ID) experiments. This document is the result of the discussions that took place during the first public meeting of the LHCDMWG [2], and it is intended to provide a template for the presentation of the LHC results at the winter conferences in 2016. It reflects the feedback obtained from the participants and in subsequent iterations with members of the experiments and of the theory community and it is based on work described recently in [3-9]. For earlier articles discussing aspects of simplified $s$-channel DM models, see also [10-21].

The relevant details of simplified DM models involving vector, axial-vector, scalar and pseudo-scalar $s$-channel mediators are first reviewed in Section 2. Section 3 presents a recommendation for the primary treatment of LHC DM bounds and introduces all of the basic assumptions entering the approach. Section 4 describes a well-defined translation procedure, including all relevant formulae and corresponding references, that allows for meaningful and fair comparisons with the limits obtained by DD and ID experiments.

\section{Models considered}

The recommendations in this proposal, adopt the model choices made for the early Run-2 LHC searches by the ATLAS/CMS DM Forum [1]. In this document we discuss models which assume that the DM particle is a Dirac fermion $\chi$ and that the particle mediating the interaction (the "mediator") is exchanged in the $s$-channel. ${ }^{1}$ After simplifying assumptions, each model is characterised by four parameters: the DM mass $m_{\mathrm{DM}}$, the mediator

\footnotetext{
1 An orthogonal set of models describe $t$-channel exchange [22-25]. This class of simplified DM models is left for future iterations and will thus not be discussed in the following.
}

mass $M_{\text {med }}$, the universal mediator coupling to quarks $g_{q}$ and the mediator coupling to DM $g_{\mathrm{DM}}$. Mediator couplings to leptons are always set to zero in order to avoid the stringent LHC bounds from di-lepton searches. In the limit of large $M_{\text {med }}$, these (and all) models converge to a universal set of operators in an effective field theory (EFT) $[13,14,26-29]$. In this section, we review the models and give the formulae for the total decay width of the mediators in each case.

\subsection{Vector and axial-vector models}

The two models with a spin-1 mediator $Z^{\prime}$, have the following interaction Lagrangians

$$
\begin{aligned}
\mathcal{L}_{\text {vector }} & =-g_{\mathrm{DM}} Z_{\mu}^{\prime} \bar{\chi} \gamma^{\mu} \chi-g_{q} \sum_{q=u, d, s, c, b, t} Z_{\mu}^{\prime} \bar{q} \gamma^{\mu} q, \\
\mathcal{L}_{\text {axial-vector }} & =-g_{\mathrm{DM}} Z_{\mu}^{\prime} \bar{\chi} \gamma^{\mu} \gamma_{5} \chi-g_{q} \sum_{q=u, d, s, c, b, t} Z_{\mu}^{\prime} \bar{q} \gamma^{\mu} \gamma_{5} q .
\end{aligned}
$$

Note that the universality of the coupling $g_{q}$ guarantees that the above spin- 1 simplified models are minimal flavour violating (MFV) [30], which is crucial to avoid the severe existing constraints arising from quark flavour physics.

The minimal decay width of the mediator is given by the sum of the partial widths for all decays into DM and quarks that are kinematically accessible. For the vector mediator, the partial widths are given by

$$
\begin{aligned}
& \Gamma_{\text {vector }}^{\chi \bar{\chi}}=\frac{g_{\mathrm{DM}}^{2} M_{\text {med }}}{12 \pi}\left(1-4 z_{\mathrm{DM}}\right)^{1 / 2}\left(1+2 z_{\mathrm{DM}}\right), \\
& \Gamma_{\text {vector }}^{q \bar{q}}=\frac{g_{q}^{2} M_{\text {med }}}{4 \pi}\left(1-4 z_{q}\right)^{1 / 2}\left(1+2 z_{q}\right),
\end{aligned}
$$

where $z_{\mathrm{DM}, q}=m_{\mathrm{DM}, q}^{2} / M_{\text {med }}^{2}$ and the two different types of contribution to the width vanish for $M_{\text {med }}<2 m_{\mathrm{DM}, q}$. The corresponding expressions for the axial-vector mediator are

$$
\begin{aligned}
\Gamma_{\text {axial-vector }}^{\chi \bar{\chi}} & =\frac{g_{\mathrm{DM}}^{2} M_{\mathrm{med}}}{12 \pi}\left(1-4 z_{\mathrm{DM}}\right)^{3 / 2}, \\
\Gamma_{\text {axial-vector }}^{q \bar{q}} & =\frac{g_{q}^{2} M_{\mathrm{med}}}{4 \pi}\left(1-4 z_{q}\right)^{3 / 2} .
\end{aligned}
$$

\subsection{Scalar and pseudo-scalar models}

The two models with a spin-0 mediator $\phi$ are described by

$$
\begin{aligned}
\mathcal{L}_{\text {scalar }} & =-g_{\mathrm{DM}} \phi \bar{\chi} \chi-g_{q} \frac{\phi}{\sqrt{2}} \sum_{q=u, d, s, c, b, t} y_{q} \bar{q} q, \\
\mathcal{L}_{\text {pseudo-scalar }} & =-i g_{\mathrm{DM}} \phi \bar{\chi} \gamma_{5} \chi-i g_{q} \frac{\phi}{\sqrt{2}} \sum_{q=u, d, s, c, b, t} y_{q} \bar{q} \gamma_{5} q,
\end{aligned}
$$

where $y_{q}=\sqrt{2} m_{q} / v$ are the SM quark Yukawa couplings with $v \simeq 246 \mathrm{GeV}$ the Higgs vacuum expectation value. These interactions are again compatible with the MFV hypothesis.

In these models, there is a third contribution to the minimal width of the mediator, which arises from loop-induced decays 
into gluons. For the scalar mediator, the individual contributions are given by

$\Gamma_{\text {scalar }}^{\chi \bar{\chi}}=\frac{g_{\text {DM }}^{2} M_{\text {med }}}{8 \pi}\left(1-4 z_{\mathrm{DM}}^{2}\right)^{3 / 2}$,

$\Gamma_{\text {scalar }}^{q \bar{q}}=\frac{3 g_{q}^{2} y_{q}^{2} M_{\text {med }}}{16 \pi}\left(1-4 z_{q}^{2}\right)^{3 / 2}$,

$\Gamma_{\text {scalar }}^{g g}=\frac{\alpha_{s}^{2} g_{q}^{2} M_{\text {med }}^{3}}{32 \pi^{3} v^{2}}\left|f_{\text {scalar }}\left(4 z_{t}\right)\right|^{2}$,

while the corresponding expressions in the pseudo-scalar case read

$$
\begin{aligned}
\Gamma_{\text {pseudo-scalar }}^{\chi \bar{x}} & =\frac{g_{\mathrm{DM}}^{2} M_{\text {med }}}{8 \pi}\left(1-4 z_{\mathrm{DM}}^{2}\right)^{1 / 2}, \\
\Gamma_{\text {pseudo-scalar }}^{q \bar{q}} & =\frac{3 g_{q}^{2} y_{q}^{2} M_{\text {med }}}{16 \pi}\left(1-4 z_{q}^{2}\right)^{1 / 2}, \\
\Gamma_{\text {pseudo-scalar }}^{g g} & =\frac{\alpha_{s}^{2} g_{q}^{2} M_{\text {med }}^{3}}{32 \pi^{3} v^{2}}\left|f_{\text {pseudo-scalar }}\left(4 z_{t}\right)\right|^{2} .
\end{aligned}
$$

Here the form factors take the form

$$
\begin{aligned}
f_{\text {scalar }}(\tau) & =\tau\left[1+(1-\tau) \arctan ^{2}\left(\frac{1}{\sqrt{\tau-1}}\right)\right], \\
f_{\text {pseudo-scalar }}(\tau) & =\tau \arctan ^{2}\left(\frac{1}{\sqrt{\tau-1}}\right) .
\end{aligned}
$$

Note that $f_{\text {scalar }}(\tau)$ and $f_{\text {pseudo-scalar }}(\tau)$ are still defined for $\tau<$ 1 , but in this case the form factors are complex. The tree-level corrections to the total widths of the mediator again do not contribute if $M_{\text {med }}<2 m_{\mathrm{DM}, q}$, meaning that the corresponding final state cannot be produced on-shell. Decays to gluon pairs are only relevant for mediator masses between roughly $200 \mathrm{GeV}$ and $400 \mathrm{GeV}$ and if invisible decays are kinematically forbidden.

\section{Presentation of $\mathrm{LHC}$ results}

The simplified DM models defined in the last section aim at capturing accurately the characteristics of MET production at high-energy colliders. They can be understood as a limit of a more general new-physics scenario, where all but the lightest dark-sector states are assumed to be sufficiently decoupled, so that only the interactions that are relevant at LHC energies are interactions between the mediator and DM as well as the SM quarks. Aside from this important caveat, a presentation of collider bounds in the simplified model framework requires no further assumptions, meaning that LHC searches can be used to set model-independent bounds on the parameter space of a simplified model, and that the constraints arising from different channels - e.g. mono-jets and di-jets - can be directly compared (see for instance [31]). In this section, we spell out the model choices underlying the LHC limits and the relic density calculations. Issues arising in the DD and ID context are discussed in subsequent sections.

\subsection{Mass-mass plane}

The advocated plots represent only two dimensional slices of the full four dimensional parameter space of the proposed simplified models. To allow for a qualitative understanding of the dependence of the results on the mediator couplings $g_{q}$ and $g_{\mathrm{DM}}$, we advocate an auxiliary figure that shows the limit on the "signal strength" $\mu$, i.e. the ratio of the experimental limit to the predicted signal cross section for fixed masses or fixed coupling scenarios. We recommend however to clarify that a limit on $\mu$ must not be confused with a bound on a rescaling factor for the couplings and thus in general cannot be used to

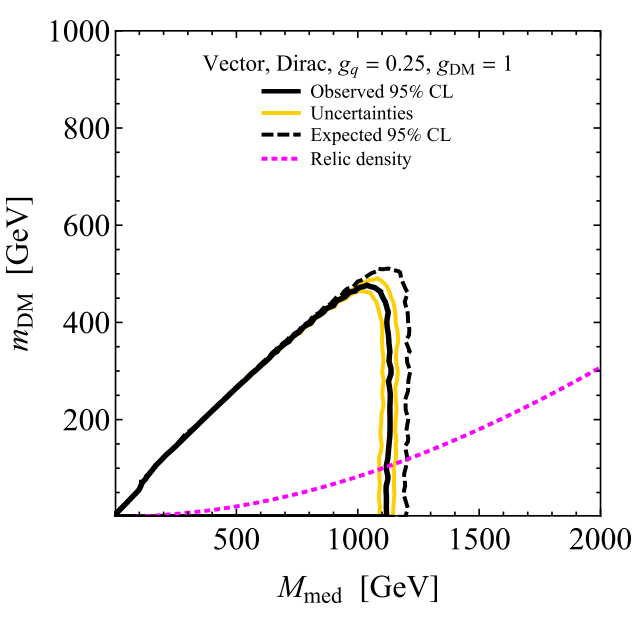

Fig. 1. $95 \% \mathrm{CL}$ exclusion contours in the mass-mass plane for a simplified model with a vector mediator, Dirac DM and couplings $g_{q}=0.25$ and $g_{D M}=1$. The black solid (dashed) curve shows the median of the observed (expected) limit, while the yellow curves indicate an example of the uncertainties on the observed bound. A minimal width is assumed and the excluded parameter space is to the bottom-left of all contours. The dotted magenta curve corresponds to the parameters where the correct DM relic abundance is obtained from standard thermal freeze-out for the chosen couplings. DM is overproduced to the bottomright of the curve. The shown LHC results are intended for illustration only and are not based on real data.

translate the exclusion limit in the mass-mass plane from one set of couplings to another. The reason is that changing $g_{q}$ and $g_{\text {DM }}$ typically modifies the total width of the mediator, which can change the kinematic distributions of the signal and thus the exclusion bounds in a non-trivial way. Furthermore, for scenarios where the mediator widths varies significantly as the function of the considered parameter (e.g. mass-mass plane), we suggest to add supporting material that illustrates the variation of the width in these parameters.

The primary presentation recommended for LHC results in the simplified model language are plots of the experimental confidence level $(\mathrm{CL})$ limits on the signal cross sections as a function of the two mass parameters $m_{\mathrm{DM}}$ and $M_{\text {med }}$ for a fixed set of couplings $g_{q}$ and $g_{\mathrm{DM}}$. An example of such a "mass-mass" plot is given in Fig. 1. It shows $95 \% \mathrm{CL}$ exclusion limits (black and yellow curves) for the case of a vector mediator. The limits are derived from a hypothetical LHC mono-jet measurement. The particular choice of axes, with $M_{\text {med }}$ on the $x$-axis and $m_{\mathrm{DM}}$ on the $y$-axis, follows the convention adopted when interpreting supersymmetry searches at the LHC. The parameter space shown in the mass-mass plots can be divided into three regions:

On-shell region: The on-shell region, $M_{\text {med }}>2 m_{\mathrm{DM}}$, is the region where LHC searches for MET signatures provide the most stringent constraints. The production rate of the mediator decreases with increasing $M_{\text {med }}$ and so does the signal strength in mono-jet searches. In this region the experimental limits and the signal cross sections depend in a complex way on all parameters of the simplified model, and it is therefore in general not possible to translate the CL limit obtained for one fixed set of couplings $g_{q}$ and $g_{\mathrm{DM}}$ to another by a simple rescaling procedure.

Off-shell region: In the off-shell region, $M_{\text {med }}<2 m_{\mathrm{DM}}$, pairproduction of DM particles turns off and the constraints from MET searches rapidly lose power. The cross sections become proportional to the combination $g_{q}^{2} g_{\mathrm{DM}}^{2}$ of couplings, so that in principle the LHC exclusions corresponding to different coupling choices can be derived by simple 
rescalings. Deviations from this scaling are observed on the interface between on-shell and off-shell regions $M_{\text {med }} \simeq$ $2 m_{\text {DM }}$ [32]. Note that for $M_{\text {med }}<2 m_{\text {DM }}$ an on-shell mediator will always decay back to SM particles, meaning that the off-shell region can be probed by non-MET searches such as di-jets or di-tops. We also note that if the mediator is light and very weakly coupled to the SM quarks, constraints from DD and/or ID on these models may be typically stronger than those from the LHC.

Heavy mediator limit: The DM EFT limit is approached as the mediator mass $M_{\text {med }}$ becomes large. In this limit the monojet cross sections scale with the fourth inverse power of the effective suppression scale $M_{*}=M_{\text {med }} / \sqrt{g_{q} g_{\text {DM }}}$. For perturbative couplings (i.e. $\sqrt{g_{q} g_{\mathrm{DM}}} \ll 4 \pi$ ), the EFT results apply to mediators with masses in the multi-TeV range.

As in the template plot, any presentation of the LHC limits has to clearly state the model assumptions made to obtain the exclusion contours. We thus advocate to explicitly specify on the figure the simplified model, including the mediator and DM type, and the choices of couplings. Besides the observed exclusion bound, the median of the expected limit and uncertainties (e.g. those arising from scale variations or ambiguities related to the choice of parton distribution functions, as well as experimental uncertainties) are useful information that can be added to these plots. All these ingredients have been included in Fig. 1.

The usefulness of the bound on $\mu$ is thus limited to cases where kinematic distributions are the same for different realisations of the simplified model. Such a situation is realised for example in the on-shell region if all couplings are sufficiently small, so that the total decay width of the mediator obeys $\Gamma_{\text {med }} \lesssim$ $0.3 M_{\text {med }}$. Under these circumstances, one can use the narrowwidth approximation (NWA) to show, for example, that in the case of a spin- 1 mediator the mono-jet cross section $\sigma(p p \rightarrow$ $\chi \bar{\chi}+j)$ factorises into mediator production $\sigma\left(p p \rightarrow Z^{\prime}+j\right)$ and the invisible branching ratio $\operatorname{Br}\left(Z^{\prime} \rightarrow \chi \bar{\chi}\right)$. This factorisation implies that a bound on $\mu$ can be used to infer a limit on the invisible branching ratio $\operatorname{Br}\left(Z^{\prime} \rightarrow \chi \bar{\chi}\right)$ of the spin-1 mediator relative to the one in the benchmark model without regenerating the underlying signal Monte Carlo (MC). Since the NWA can be an imperfect approximation even for weak couplings $g_{q}$ and $g_{\mathrm{DM}}$ (see for instance [32]), we recommend that care is taken if relying on this argument.

If readers would like to reinterpret experimental limits for different coupling choices, it is their responsibility to ensure that kinematic distributions remain unchanged. To make this issue clear, we recommend that captions of plots showing limits on $\mu$ include a statement along the lines of "Note that the bound on $\mu$ only applies to coupling combinations that yield the same kinematic distributions as the benchmark model".

\subsection{Choice of couplings for presentation of results in mass-mass plane}

At present, we recommend that mono-jet-like searches produce limits for a single choice of couplings. The ATLAS/CMS DM Forum report [1] forms the basis of our recommendations for the simplified models given in Section 2. In particular, we advocate the following coupling values to produce the limits on signal strengths:

Vector mediator: $g_{\mathrm{DM}}=1$ and $g_{q}=0.25$.

Axial-vector mediator: $g_{\mathrm{DM}}=1$ and $g_{q}=0.25$

Scalar mediator: $g_{q}=1$ and $g_{\mathrm{DM}}=1$.
Pseudo-scalar mediator: $g_{q}=1$ and $g_{\mathrm{DM}}=1$.

The quark coupling $g_{q}$ should be universal in all cases and the width of the mediator should be set to the minimal width, meaning that it is assumed that the mediator has no couplings other than $g_{q}$ and $g_{\mathrm{DM}}{ }^{2}$. The choices above provide for a consistent comparison across collider results within a given simplified model. They ensure that the mediator has $\Gamma_{\text {med }} / M_{\text {med }} \lesssim 10 \%$ and that the theory is far from the strong coupling regime. The choice of $g_{q}=0.25$ for spin- 1 mediators is furthermore motivated by the requirement to avoid di-jet constraints from the LHC and earlier hadron colliders (see e.g. [31]). When readers are interested in extrapolating the provided results to other coupling values, it is their responsibility to understand how changing $g_{q}$ and $g_{\mathrm{DM}}$ will affect the kinematics of the signal and therefore the experimental CL limits. To facilitate such an extrapolation, ATLAS and CMS could provide additional information (e.g. tables of acceptances, efficiencies, number of events generated, total experimental uncertainty, number of events passing analysis cuts for benchmark signals) corresponding to the recommended coupling choices as supplementary material, as detailed in Appendix B of [1]. As discussed in [1], the kinematics of vector and axial-vector models is very similar in the case of jet radiation. The same consideration applies for the scalar and pseudo-scalar models in the mono-jet channel, while differences are seen for heavy flavour final states.

\subsection{Overlaying additional information on LHC results}

Fixing both $g_{q}$ and $g_{\mathrm{DM}}$ has the advantage that, in a given model, one can compare the LHC results to relic density calculations or the limits obtained by DD and ID experiments. Nevertheless, such comparisons typically require additional assumptions and should be done carefully. We discuss a few possibilities below. In all cases, we recommend to keep the plots simple, and to specify the assumptions clearly or to produce several variations to indicate the impact that different assumptions have on the final results.

\subsubsection{Relic density}

Relic density calculations can be overlaid on the mass-mass plot to indicate where the particles and interactions of a specific simplified model are by themselves sufficient for explaining the observed DM abundance. For the simplified models recommended by the ATLAS/CMS DM Forum, this curve corresponds to the parameters for which the observed relic abundance is compatible with a single species of DM Dirac fermion and a single mediator that couples to all SM quarks with equal strength. One should not conclude that a simplified model is ruled out for values of model parameters that are inconsistent with the relic density overlay. Rather, one should conclude that additional physics beyond the simplified model was relevant for determining the DM abundance in the early Universe.

When calculating the relic density, we recommend to include all tree-level processes relevant for the DM annihilation. In particular, when $M_{\text {med }}<m_{\mathrm{DM}}$, annihilation into on-shell mediators are typically active, and are particularly important when $g_{\mathrm{DM}} \gg g_{q}$ (e.g. [21]), for which cross sections are typically insensitive to $g_{q}$, unlike LHC processes.

\footnotetext{
2 Using the same value of $g_{q}$ for all quarks is theoretically well motivated for the vector, scalar and pseudo-scalar mediator. For the axial-vector mediator it would also be interesting to consider $g_{u}=g_{c}=g_{t}=-g_{d}=-g_{s}=-g_{b}$, which arises naturally if the vector mediator corresponds to the massive gauge boson of a new broken $U(1)^{\prime}$ and the SM Yukawa couplings are required to be invariant under this additional gauge symmetry. The relative sign between the coupling to up-type and down-type quarks is important if interference plays a role and affects the comparison between LHC and DD results.
} 
Numerical tools, such as micrOMEGAs [33] and MadDM [34], can be used to calculate the regions of relic overproduction or underproduction for the simplified models recommended by the ATLAS/CMS DM Forum. We provide the results of MadDM calculations for the models described in Section 2 at [35]. These results were obtained using the coupling values specified in Section 3.2. The reader should be aware that the axial-vector calculation does not include an explicit constraint from perturbative unitarity (described below). The provided curves correspond to $\Omega_{\chi} h^{2}=0.12$ (the relic DM density observed by WMAP [36] and Planck [37]) for the models considered. Larger mediator masses as well as smaller DM masses (below the curves) correspond to larger values of $\Omega_{\chi} h^{2}$ (and conversely for smaller mediator masses and larger DM masses).

\subsubsection{Perturbativity limits, anomalies and issues with gauge invari-} ance

The couplings recommended by the ATLAS/CMS DM Forum have been fixed to values which are perturbative, with the mediator width always sufficiently smaller than the mediator mass. However, it was shown in $[31,38$ ] that perturbative unitarity is violated in the axial-vector model due to the DM Yukawa coupling becoming non-perturbative, even for perturbative values of $g_{q}$ and $g_{\mathrm{DM}}$, if $m_{\mathrm{DM}}$ is significantly larger than $M_{\text {med. }}$. It was argued that this consideration implies $m_{\mathrm{DM}}^{2} g_{\mathrm{DM}}^{2} /\left(\pi M_{\text {med }}^{2}\right)<1 / 2$, which yields $m_{\mathrm{DM}}<\sqrt{\pi / 2} M_{\text {med }}$ for the recommended value $g_{\mathrm{DM}}=1$. It is therefore proposed to indicate the line corresponding to $m_{\mathrm{DM}}=\sqrt{\pi / 2} M_{\text {med }}$ in the mass-mass plot for the axial-vector case in a similar style as for the relic density constraint (i.e. just a line, no shading).

Another potential problem of the vector and axial-vector model is that they are not anomaly free if the $Z^{\prime}$ boson couples only to quarks but not to leptons. This implies that the full theory that ultraviolet completes (1) and (2) must include new fermions to cancel the anomalies. While these fermions can be vector-like with respect to the SM, they will need to be chiral with respect to the new gauge group that gives rise to the $Z^{\prime}$. In consequence, the additional fermions must have masses of the order of the symmetry-breaking scale, which is at most a factor of a few above $M_{\text {med }}$ [38]. While the existence of additional fermions will lead to new signatures, the precise impact on LHC phenomenology depends on the specific way the anomalies are cancelled. The resulting model dependence is difficult to quantify and we thus propose to ignore the issue of anomalies until it has been studied in detail by theorists.

The interactions between the spin- 0 mediator and the quarks present in the simplified scalar model are not $S U(2)_{L}$ invariant. As a result, these interactions will violate perturbative unitarity at high energies in tree-level process like $p p \rightarrow W+\phi(\phi \rightarrow$ $\chi \bar{\chi})$. The corresponding amplitudes are however proportional to the squares of the light-quark Yukawa couplings, so that in practice unitarity-violating effects are expected to have a negligible impact on the outcome of MET searches at the LHC. Still in $S U(2)_{L}$ invariant theories that provide specific realisation of the $s$-channel scalar mediator interactions (7), like for instance the fermion singlet DM model (see e.g. [9]), the resulting LHC phenomenology can be modified by the new fields that are needed to make the full theory gauge invariant. These modifications are again model dependent and lacking detailed theoretical studies, their effect on the LHC bounds cannot yet be quantified.

\subsubsection{Additional plots}

Above, we recommend that LHC searches present the massmass plot, fixing both $g_{q}$ and $g_{\mathrm{DM}}$, as the primary result. If desired, additional information on the coupling dependence of the results can be conveyed by producing a related set of limits where one of the mass parameters and one of the couplings has been fixed, and the other mass parameter and coupling are varied. As discussed in the previous section, a correct treatment of varying couplings is one which correctly accounts for the varying acceptance of the search.

\subsubsection{Non-collider DM searches}

Interpreting non-collider results in the simplified model framework involves additional assumptions, and generally requires detailed knowledge of how the non-collider results were produced. For example, as discussed above, the relic density predicted by the simplified model varies from point to point on the mass-mass plot, whereas non-collider results are typically presented under the assumption that the density of the DM particle under consideration saturates the cosmological density (i.e. that there is just one species of DM). These assumptions may be consistent if there is additional physics (not captured by the simplified model) that affects the relic density calculation but is irrelevant to the LHC signals (see e.g. [39]). However, it is also a possibility that the DM particle probed by non-collider experiments constitutes only a certain component of the DM density, so their results would have to be rescaled accordingly (see for instance [31]). Because of the ambiguity of this rescaling, we do not recommend mapping from non-collider results onto the LHC mass-mass plots. The following section addresses the comparison of LHC and non-collider results.

\section{Comparison to non-collider results}

Although we advocate mass-mass plots as the primary presentation of LHC results, it is nevertheless interesting and informative to compare the LHC limits with the results from other DM searches. To avoid the difficulties associated with reinterpreting the results of non-collider experiments, we recommend translating the LHC results onto the plots of non-collider experiments, rather than the reverse procedure. When performing a translation to the non-collider planes, it is important to bear in mind the different underlying assumptions. While the DD or ID bounds may be valid for multiple DM models, the LHC limits hold exclusively for the mediator under investigation and for the specific choices of the couplings used in the simplified model.

For a given mediator the translation procedure is well-defined. In this section, we explain all of the ingredients needed for a correct translation into the cross section-mass planes in which DD and ID experiments present their results. As input, we use LHC bounds in the mass-mass plane for fixed couplings $g_{q}$ and $g_{\mathrm{DM}}$ (see Section 3). To compare with DD experiments, these limits are translated into the planes of the DM mass $m_{\mathrm{DM}}$ versus the spin-independent (SI) or spin-dependent (SD) DM-nucleon cross section, $\sigma_{\mathrm{SI}}$ or $\sigma_{\mathrm{SD}}$. For a comparison with ID experiments, the limits are instead converted into the plane defined by $m_{\mathrm{DM}}$ and the DM annihilation cross section $\left\langle\sigma v_{\text {rel }}\right\rangle$.

\subsection{DD experiments}

DD experiments search for the recoil of a nucleus scattering off a DM particle traversing the detector. Since the DM particle is non-relativistic, the dominant interactions between DM and nuclei can be described by two effective parameters, namely the SI and SD DM-nucleon scattering cross sections. DD experiments present their limits as bounds on these cross section as a function of $m_{\mathrm{DM}}$, where common units for the cross section are either $\mathrm{cm}^{2}$ or $\mathrm{pb}$. The bounds are presented at $90 \% \mathrm{CL}$, as opposed to the 95\% CL limits that are the standard in the collider community. For the sake of comparison, we recommend to present the LHC limits on the $m_{\mathrm{DM}}-\sigma_{\mathrm{SI} / \mathrm{SD}}$ planes at $90 \% \mathrm{CL}$. 
In principle, it is necessary to distinguish between the DMproton scattering cross sections $\sigma_{\mathrm{SI} / \mathrm{SD}}^{p}$ and the DM-neutron scattering cross sections $\sigma_{\mathrm{SI} / \mathrm{SD}}^{n}$. For SI interactions, however, DD bounds are always shown under the assumption that $\sigma_{\mathrm{SI}}^{p}=\sigma_{\mathrm{SI}}^{n}$, which also holds for the simplified models proposed here. For SD interactions it is common to present separate bounds on $\sigma_{\mathrm{SD}}^{p}$ and $\sigma_{\mathrm{SD}}^{n}$ and it is possible to compare LHC results with both.

There are currently a rather large number of DD experiments that have different target nuclei and use different detection technologies. For SI interactions, the most sensitive experiments for DM particles heavier than $\mathcal{O}(10 \mathrm{GeV})$ are two-phase xenon experiments. There are two large competing collaborations employing this technology, LUX [40] and XENON1T [41] (previously XENON100). LUX has published results from its first run and is currently collecting more data to improve its sensitivity. XENON1T will soon begin its first run and aims to have first results in late 2016. For DM particles lighter than $\mathcal{O}(10 \mathrm{GeV})$, solid state cryogenic detectors as used by the SuperCDMS [42] and CRESST-II [43] collaborations are more constraining than xenon experiments as their energy threshold is lower.

As mentioned above, for SD interactions, separate bounds are published on $\sigma_{\mathrm{SD}}^{p}$ and $\sigma_{\mathrm{SD}}^{n}$. This is because DM scatters with the spin of the isotope which is approximately due to an unpaired neutron or unpaired proton. In practice this means that DD experiments have good sensitivity to $\sigma_{\mathrm{SD}}^{p}$ or $\sigma_{\mathrm{SD}}^{n}$ but not both. The strongest DD limits on $\sigma_{\mathrm{SD}}^{p}$ are from the PICO collaboration [44, 45], while the strongest limits on $\sigma_{\mathrm{SD}}^{n}$ are from LUX [46]. ${ }^{3}$

The simplified models with a vector and scalar mediator lead to a SI interaction, while the axial-vector and pseudo-scalar mediator induce SD interactions. The pseudo-scalar interaction has additional velocity-suppression in the non-relativistic limit, which is not present in the other interactions. In practice this means that pseudo-scalar interactions are only very weakly testable with DD experiments. For this reason, we will only describe the translation procedure into the $m_{\mathrm{DM}}-\sigma_{\mathrm{SI} / \mathrm{SD}}$ plane for vector, axial-vector and scalar interactions.

Sections 4.1 .1 and 4.1.2 detail procedures for translating LHC limits onto to the $m_{\mathrm{DM}}-\sigma_{\mathrm{SI} / \mathrm{SD}}$ planes. Figs. $2 \mathrm{a}$ and $2 \mathrm{~b}$ illustrate the conventions recommended for the presentation of results obtained from these procedures. These plots show the minimum number of DD limits that we recommend to show. Bounds from other experiments may also be included. As in the mass-mass plots, we recommend to explicitly specify details of the mediator and DM type, the choices of couplings and the CL of the exclusion limits. It may also be useful to show theoretical and experimental uncertainties. Generally, the LHC searches exclude the on-shell region in the mass-mass plane such that for a fixed value of $m_{\mathrm{DM}}$, the exclusion contour passes through two values of $M_{\text {med }}$. This means that when translating into the $m_{\mathrm{DM}}-\sigma_{\mathrm{SI} / \mathrm{SD}}$ planes, for a fixed value of $m_{\mathrm{DM}}$, the exclusion contour must pass through two values of $\sigma_{\mathrm{SI} / \mathrm{SD}}$. This explains the turnover behaviour of the LHC contours observed in Figs. 2a and 2b.

\subsubsection{SI cases: Vector and scalar mediators}

In general, the SI DM-nucleon scattering cross section takes the form

$\sigma_{\mathrm{SI}}=\frac{f^{2}\left(g_{q}\right) g_{\mathrm{DM}}^{2} \mu_{n \chi}^{2}}{\pi M_{\mathrm{med}}^{4}}$,

where $\mu_{n \chi}=m_{n} m_{\mathrm{DM}} /\left(m_{n}+m_{\mathrm{DM}}\right)$ is the DM-nucleon reduced mass with $m_{n} \simeq 0.939 \mathrm{GeV}$ the nucleon mass. The mediatornucleon coupling is $f\left(g_{q}\right)$ and depends on the mediator-quark

\footnotetext{
3 Note an open source data resource where many DD experiments have uploaded limits is DMTools [47]. These data, however, are not always officially blessed or scrutinised by the experiments and thus should be used with care.
}

couplings. For the interactions mediated by vector and scalar particles and for the recommended coupling choices, the difference between the proton and neutron cross section is negligible.

For the vector mediator,

$f\left(g_{q}\right)=3 g_{q}$,

and hence

$\sigma_{\mathrm{SI}} \simeq 6.9 \times 10^{-41} \mathrm{~cm}^{2} \cdot\left(\frac{g_{q} g_{\mathrm{DM}}}{0.25}\right)^{2}\left(\frac{1 \mathrm{TeV}}{M_{\text {med }}}\right)^{4}\left(\frac{\mu_{n \chi}}{1 \mathrm{GeV}}\right)^{2}$.

For the simplified model with scalar mediator exchange we follow the recommendation of ATLAS/CMS DM Forum [1] and assume that the scalar mediator couples to all quarks (like e.g. the SM Higgs). In general the formula for $f\left(g_{q}\right)$ is

$f^{n, p}\left(g_{q}\right)=\frac{m_{n}}{v}\left[\sum_{q=u, d, s} f_{q}^{n, p} g_{q}+\frac{2}{27} f_{\mathrm{TG}}^{n, p} \sum_{Q=c, b, t} g_{Q}\right]$.

Here $f_{\mathrm{TG}}^{n, p}=1-\sum_{q=u, d, s} f_{q}^{n, p}$. The state-of-the-art values for $f_{q}^{n, p}$ are from [48] (for $f_{u}^{n, p}$ and $f_{d}^{n, p}$ ) and [49] (for $f_{s}^{n, p}$ ) and read $f_{u}^{n}=0.019, f_{d}^{n}=0.045$ and $f_{s}^{n}=0.043$. The values for the proton are slightly different, but in practice the difference can be ignored. Substituting these values, we find that numerically

$f\left(g_{q}\right)=1.16 \cdot 10^{-3} g_{q}$,

and therefore the size of a typical cross section is

$\sigma_{\mathrm{SI}} \simeq 6.9 \times 10^{-43} \mathrm{~cm}^{2} \cdot\left(\frac{g_{q} g_{\mathrm{DM}}}{1}\right)^{2}\left(\frac{125 \mathrm{GeV}}{M_{\text {med }}}\right)^{4}\left(\frac{\mu_{n \chi}}{1 \mathrm{GeV}}\right)^{2}$.

\subsubsection{SD case: Axial-vector mediator}

For the axial-vector mediator, the scattering is SD and the corresponding cross section can be written as

$\sigma_{\mathrm{SD}}=\frac{3 f^{2}\left(g_{q}\right) g_{\mathrm{DM}}^{2} \mu_{n \chi}^{2}}{\pi M_{\mathrm{med}}^{4}}$.

In general $f^{p, n}\left(g_{q}\right)$ differs for protons and neutrons and is given by

$f^{p, n}\left(g_{q}\right)=\Delta_{u}^{(p, n)} g_{u}+\Delta_{d}^{(p, n)} g_{d}+\Delta_{s}^{(p, n)} g_{s}$,

where $\Delta_{u}^{(p)}=\Delta_{d}^{(n)}=0.84, \Delta_{d}^{(p)}=\Delta_{u}^{(n)}=-0.43$ and $\Delta_{s}=-0.09$ are the values recommended by the Particle Data Group [50]. Other values are also used in the literature (see e.g. [51]) and differ by up to $\mathcal{O}(5 \%)$.

Under the assumption that the coupling $g_{q}$ is equal for all quarks, one finds

$f\left(g_{q}\right)=0.32 g_{q}$

and thus

$\sigma^{\mathrm{SD}} \simeq 2.4 \times 10^{-42} \mathrm{~cm}^{2} \cdot\left(\frac{g_{q} g_{\mathrm{DM}}}{0.25}\right)^{2}\left(\frac{1 \mathrm{TeV}}{M_{\text {med }}}\right)^{4}\left(\frac{\mu_{n \chi}}{1 \mathrm{GeV}}\right)^{2}$.

We emphasise that the same result is obtained both for the SD DM-proton scattering cross section $\sigma_{\mathrm{SD}}^{p}$ and the SD DM-neutron scattering cross section $\sigma_{\mathrm{SD}}^{n}$. Using (26) it is therefore possible to map collider results on both parameter planes conventionally shown by DD experiments. Should only one plot be required, we recommend comparing the LHC results to the DD bounds on $\sigma_{\mathrm{SD}}^{p}$, which is typically more difficult to constrain.

In the future, it is desirable to consider not only the case $g_{u}=g_{d}=g_{s}$, but also the case $g_{u}=-g_{d}=-g_{s}$, which is wellmotivated from embedding the simplified model in the SM gauge group and can be included without much additional effort. For $g_{u}=-g_{d}=-g_{s}$ one obtains approximately $f^{p}\left(g_{q}\right)=1.36 g_{u}$ and 


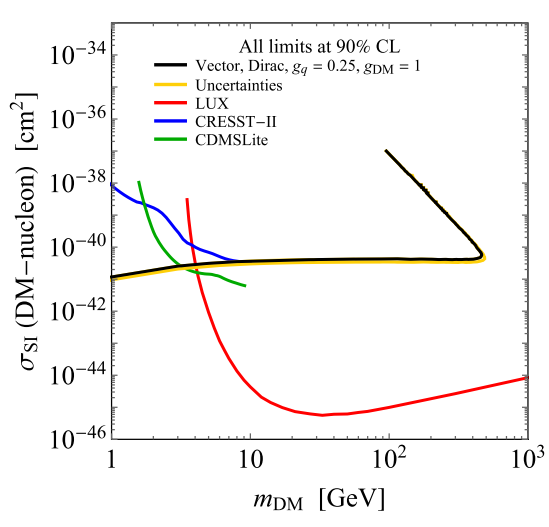

(a)

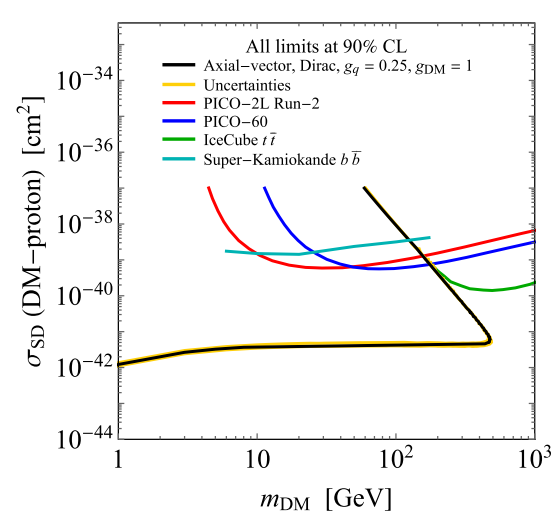

(b)

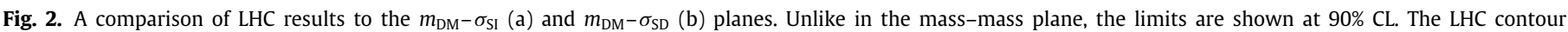

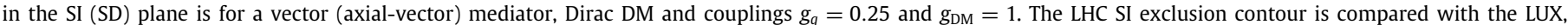

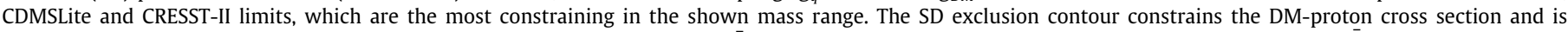

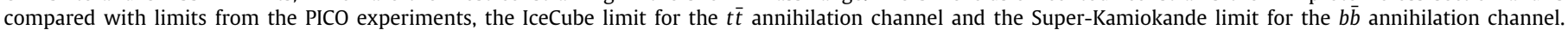
The depicted LHC results are intended for illustration only and are not based on real data.

$f^{n}\left(g_{q}\right)=-1.18 g_{u}$, i.e. the DM-neutron cross section is slightly smaller than the DM-proton cross section. ${ }^{4}$

\subsubsection{Neutrino observatories: IceCube and Super-Kamiokande}

The IceCube [53] and Super-Kamiokande [54] neutrino observatories are also able to constrain the SI and SD cross sections. When DM particles elastically scatter with elements in the Sun, they can lose enough energy to become gravitationally bound. Self-annihilation of the DM particles produces neutrinos (either directly or in showering) that can be searched for in a neutrino observatory. When the DM capture and annihilation rates are in equilibrium, the neutrino flux depends only on the initial capture rate, which is determined by the SI or SD cross section [55].

The IceCube and Super-Kamiokande limits on $\sigma_{\mathrm{SD}}^{p}$ are of particular interest as they can be stronger than the corresponding bounds from DD experiments. The former bounds are however more model dependent, since they depend on the particular DM annihilation channel. For annihilation only into light quarks, the limits are weaker than DD experiments. For $m_{b}<m_{\mathrm{DM}}<m_{t}$, on the other hand, the dominant annihilation channel of the axialvector model is to $b \bar{b}$ and Super-Kamiokande sets more stringent constraints than DD experiments for $m_{\mathrm{DM}}<10 \mathrm{GeV}$. For $m_{\mathrm{DM}}>$ $m_{t}$, the dominant annihilation channel is to $t \bar{t}$ and the resulting constraints from IceCube are stronger than DD experiments. Both the Super-Kamiokande and IceCube limits can be shown together with other bounds on the SD DM-proton scattering cross section.

While strong bounds are obtained for annihilation into bosons or leptons, these couplings are not present in the simplified models considered here. Therefore, we do not recommend showing the IceCube or Super-Kamiokande limits for annihilation into bosons or leptons. Note also that the IceCube bounds may be further modified if the DM particles can directly annihilate into the mediator (see the discussion in [56]). For $m_{\mathrm{DM}} \lesssim 4 \mathrm{GeV}$, the effects of DM evaporation from the Sun are important, so placing limits on $\sigma_{\mathrm{SD}}^{p}$ and $\sigma_{\mathrm{SI}}$ from neutrinos coming from the Sun becomes very difficult in this low-mass regime (see e.g. [57]).

\footnotetext{
4 LHC searches are only sensitive to the relative sign between $g_{u}$ and $g_{d}$ if both types of quarks are present in a single process (e.g. $u \bar{d} \rightarrow u \bar{d}+\chi \bar{\chi}$ or $u \bar{u} \rightarrow d \bar{d}+\chi \bar{\chi})$. Such processes give a subleading effect in mono-jet searches and are presently not included in the signal computation. As a result, the signal prediction for mono-jets turns out to be independent of the relative sign between the individual quark couplings [52].
}

\subsection{ID experiments}

For a pseudo-scalar mediator, the rate at DD experiments is suppressed by additional velocity-dependent terms entering the cross section. As a result, DD experiments have very little sensitivity for this scenario and it is not worthwhile to compare LHC results to the usual bounds on SI and SD cross sections. Instead, LHC bounds can be compared against the limits from ID experiments. For example, Fermi-LAT places 95\% CL constraints on the self-annihilation cross section from observations of dwarf spheroidal galaxies [58]..$^{5}$ Limits are set on the cross section $\left\langle\sigma v_{\text {rel }}\right\rangle$ to annihilate to a single particle-anti-particle final state.

There are a number of subtleties when dealing with these limits. Firstly, all of the bounds shown in [58] are for a Majorana fermion. ID annihilation cross section limits for a Dirac fermion are larger by a factor of two and therefore need to be rescaled before they can be compared to the Dirac DM simplified model considered here. Secondly, the limits are for single particle-antiparticle final states while models typically include more than one final state. For the pseudo-scalar model, for example, DM annihilates to all quarks with branching ratios approximately proportional to $m_{q}^{2}$. In practice, however, the gamma-ray flux that is observed from annihilating to different quarks (or gluons) is small [60]. The Fermi-LAT limits [58] also demonstrate that there is a negligible difference between the limits on $\left\langle\sigma v_{\text {rel }}\right\rangle$ in $u \bar{u}$ and $b \bar{b}$ final states. We therefore suggest to only show the bound on $u \bar{u}$ from Fermi-LAT in comparison with the calculated bound on the total annihilation cross section, as representative of the limits to final states involving linear combinations of different quarks or gluons.

The annihilation cross section into a $q \bar{q}$ final state is (see e.g. [61] for a recent example)

$\left\langle\sigma v_{\mathrm{rel}}\right\rangle_{q}=\frac{3 m_{q}^{2}}{2 \pi v^{2}} \frac{g_{q}^{2} g_{\mathrm{DM}}^{2} m_{\mathrm{DM}}^{2}}{\left(M_{\mathrm{med}}^{2}-4 m_{\mathrm{DM}}^{2}\right)^{2}+M_{\mathrm{med}}^{2} \Gamma_{\mathrm{med}}^{2}} \sqrt{1-\frac{m_{q}^{2}}{m_{\mathrm{DM}}^{2}}}$,

where $\Gamma_{\text {med }}$ is the total width of the mediator (see Section 2.2). Similarly, the annihilation cross section into a pair of gluons is

5 The galactic centre is also potentially a promising DM target. Current observations show an excess of gamma rays which are roughly consistent with a DM signal, but cannot be conclusively identified as such due to poorly understood astrophysical backgrounds [59]. The regions of simplified models capable of reproducing this excess are currently regions of particular interest for collider and direct searches. 


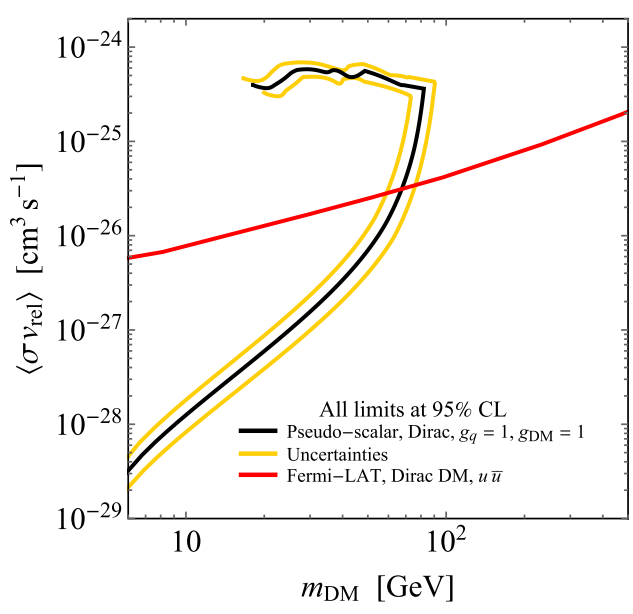

Fig. 3. A comparison of the LHC result to the Fermi-LAT limit in the $m_{\mathrm{DM}}-$ $\left\langle\sigma v_{\text {rel }}\right\rangle$ plane. Both limits are at 95\% CL. The Fermi-LAT limit is for Dirac DM and assumes that the only annihilation channel is to $u \bar{u}$ quarks. The FermiLAT limits to other quark-anti-quark annihilation channels will be similar. The LHC exclusion contour is for a pseudo-scalar mediator, Dirac DM and couplings $g_{q}=1$ and $g_{\mathrm{DM}}=1$. The shown LHC results are intended for illustration only and are not based on real data.

given by

$$
\begin{aligned}
\left\langle\sigma v_{\text {rel }}\right\rangle_{g}= & \frac{\alpha_{s}^{2}}{2 \pi^{3} v^{2}} \frac{g_{q}^{2} g_{\text {DM }}^{2}}{\left(M_{\text {med }}^{2}-4 m_{\mathrm{DM}}^{2}\right)^{2}+M_{\text {med }}^{2} \Gamma_{\text {med }}^{2}} \\
& \times\left|\sum_{q} m_{q}^{2} f_{\text {pseudo-scalar }}\left(\frac{m_{q}^{2}}{m_{\times}^{2}}\right)\right|^{2},
\end{aligned}
$$

where $f_{\text {pseudo-scalar }}(\tau)$ has been defined in (16) and $\alpha_{s}$ is the strong coupling constant, which we recommend to evaluate at the scale $\mu=2 m_{\mathrm{DM}}$. The total cross section is then given by the sum of the quark and gluon channels (27) and (28) as well as any annihilation channels into on-shell mediators which are kinematically allowed and are not suppressed by the small relative velocities of DM in the galactic halo.

Fig. 3 depicts the translation of LHC bounds for a pseudo-scalar mediator to the $m_{\mathrm{DM}}-\left\langle\sigma v_{\text {rel }}\right\rangle$ plane. As with the other plots, we recommend to specify explicitly details including the mediator and DM type, the choices of couplings and the CL of the exclusion limits. It is also important to emphasise that the ID limit is for Dirac DM instead of Majorana DM as assumed in the Fermi-LAT publication. Since the LHC exclusion contour in the mass-mass plane passes through two values of $M_{\text {med }}$, the LHC limit shows a similar turnover behaviour in the $m_{\mathrm{DM}}-\left\langle\sigma v_{\text {rel }}\right\rangle$ plane. In Fig. 3 we have depicted both branches of the exclusion contour that are obtained for fixed DM mass $m_{\mathrm{DM}}$. It may also be desirable to show the values of $\left\langle\sigma v_{\text {rel }}\right\rangle$ in Fig. 3 that produce the observed relic density. A standard reference providing the values of $\left\langle\sigma v_{\text {rel }}\right\rangle$ as a function of $m_{\mathrm{DM}}$ is [62]. We reemphasise the point made in [62] that their displayed values of $\left\langle\sigma v_{\text {rel }}\right\rangle$ should be multiplied by a factor of two for Dirac DM.

To conclude this section, we emphasise that translating DD or ID searches into bounds on the DM-nucleon scattering cross section or the DM self-annihilation cross section plane always require an assumption on the density of DM particles. In particular, it is always assumed that the particle under consideration constitutes all of the DM in the Universe. If $\chi$ is only one out of several DM sub-components, bounds from DD and ID experiments would become weaker, while the LHC bounds remain unchanged.

\section{Acknowledgements}

This document is part of a project that has received funding from the European Research Council (ERC) under the European Union's Horizon 2020 research and innovation programme (grant agreement No 679305). The work of $A B$ is partially supported by the Department of Energy Award DE-SC0011726. The work of OB is supported in part by the London Centre for Terauniverse Studies (LCTS), using funding from the European Research Council via the Advanced Investigator Grant 267352. UH acknowledges the hospitality and support of the CERN theory division. SK is supported by the New Frontiers program of the Austrian Academy of Sciences. The work of CM is part of the research programme of the Foundation for Fundamental Research on Matter (FOM), which is part of the Netherlands Organisation for Scientific Research (NWO). TMPT acknowledges support from National Science Foundation (NSF) grant PHY-1316792 and UCI through a Chancellor's Fellowship.

\section{References}

[1] D. Abercrombie, et al., Dark matter benchmark models for early LHC run-2 searches: Report of the ATLAS/CMS dark matter forum, Phys. Dark Universe (2015) http://dx.doi.org/10.1016/j.dark.2019.100371, in this issue.

[2] LHC Dark Matter WG public meeting, CERN, https://cds.cern.ch/record/ 2114807, 2015.

[3] O. Buchmueller, M.J. Dolan, S.A. Malik, C. McCabe, Characterising dark matter searches at colliders and direct detection experiments: Vector mediators, J. High Energy Phys. 01 (2015) 037, http://dx.doi.org/10.1007/ JHEP01(2015)037, arXiv:1407.8257.

[4] J. Abdallah, et al., Simplified Models for Dark Matter and Missing Energy Searches at the LHC, arXiv:1409.2893, 2014

[5] S.A. Malik, et al., Interplay and characterization of dark matter searches at colliders and in direct detection experiments, Phys. Dark Univ. 9-10 (2015) 51-58, http://dx.doi.org/10.1016/j.dark.2015.03.003, arXiv:1409.4075.

[6] M.R. Buckley, D. Feld, D. Goncalves, Scalar simplified models for dark matter, Phys. Rev. D91 (1) (2015) 015017, http://dx.doi.org/10.1103/PhysRevD. 91.015017, arXiv:1410.6497.

[7] P. Harris, V.V. Khoze, M. Spannowsky, C. Williams, Constraining dark sectors at colliders: Beyond the effective theory approach, Phys. Rev. D91 (2015) 055009, http://dx.doi.org/10.1103/PhysRevD.91.055009, arXiv: 1411.0535.

[8] U. Haisch, E. Re, Simplified dark matter top-quark interactions at the LHC, J. High Energy Phys. 06 (2015) 078, http://dx.doi.org/10.1007/JHEP06(2015) 078, arXiv:1503.00691.

[9] J. Abdallah, et al., Simplified models for dark matter searches at the LHC Phys. Dark Univ. 9-10 (2015) 8-23, http://dx.doi.org/10.1016/j.dark.2015 08.001, arXiv:1506.03116.

[10] F.J. Petriello, S. Quackenbush, K.M. Zurek, The invisible $Z^{\prime}$ at the CERN LHC, Phys. Rev. D77 (2008) 115020, http://dx.doi.org/10.1103/PhysRevD. 77.115020, arXiv:0803.4005.

[11] Y. Gershtein, F. Petriello, S. Quackenbush, K.M. Zurek, Discovering hidden sectors with mono-photon $Z^{\prime}$ searches, Phys. Rev. D78 (2008) 095002, http://dx.doi.org/10.1103/PhysRevD.78.095002, arXiv:0809.2849.

[12] E. Dudas, Y. Mambrini, S. Pokorski, A. Romagnoni, (In)visible $Z^{\prime}$ and dark matter, J. High Energy Phys. 08 (2009) 014, http://dx.doi.org/10.1088/11266708/2009/08/014, arXiv:0904.1745.

[13] Y. Bai, P.J. Fox, R. Harnik, The tevatron at the frontier of dark matter direct detection, J. High Energy Phys. 1012 (2010) 048, http://dx.doi.org/10.1007/ JHEP12(2010)048, arXiv:1005.3797.

[14] P.J. Fox, R. Harnik, J. Kopp, Y. Tsai, Missing energy signatures of dark matter at the LHC, Phys. Rev. D85 (2012) 056011, http://dx.doi.org/10. 1103/PhysRevD.85.056011, arXiv:1109.4398.

[15] J. Goodman, W. Shepherd, LHC Bounds on UV-Complete Models of Dark Matter, arXiv:1111.2359, 2011.

[16] H. An, X. Ji, L.-T. Wang, Light dark matter and $Z^{\prime}$ dark force at colliders, J. High Energy Phys. 07 (2012) 182, http://dx.doi.org/10.1007/JHEP07(2012) 182, arXiv:1202.2894.

[17] M.T. Frandsen, F. Kahlhoefer, A. Preston, S. Sarkar, K. Schmidt-Hoberg, LHC and tevatron bounds on the dark matter direct detection crosssection for vector mediators, J. High Energy Phys. 07 (2012) 123, http: //dx.doi.org/10.1007/JHEP07(2012)123, arXiv:1204.3839.

[18] H. Dreiner, D. Schmeier, J. Tattersall, Contact interactions probe effective dark matter models at the LHC, Europhys. Lett. 102 (2013) 51001, http: //dx.doi.org/10.1209/0295-5075/102/51001, arXiv:1303.3348. 
[19] R.C. Cotta, A. Rajaraman, T.M.P. Tait, A.M. Wijangco, Particle physics implications and constraints on dark matter interpretations of the CDMS signal, Phys. Rev. D90 (2014) 013020, http://dx.doi.org/10.1103/PhysRevD. 90.013020, arXiv: 1305.6609.

[20] O. Buchmueller, M.J. Dolan, C. McCabe, Beyond effective field theory for dark matter searches at the LHC, J. High Energy Phys. 01 (2014) 025, http://dx.doi.org/10.1007/JHEP01(2014)025, arXiv:1308.6799.

[21] M. Abdullah, A. DiFranzo, A. Rajaraman, T.M.P. Tait, P. Tanedo, A.M. Wijangco, Hidden on-shell mediators for the galactic center $\gamma$-ray excess, Phys. Rev. D90 (2014) 035004, http://dx.doi.org/10.1103/PhysRevD. 90.035004, arXiv:1404.6528.

[22] S. Chang, R. Edezhath, J. Hutchinson, M. Luty, Effective WIMPs, Phys. Rev. D89 (2014) 015011, http://dx.doi.org/10.1103/PhysRevD.89.015011, arXiv: 1307.8120.

[23] H. An, L.-T. Wang, H. Zhang, Dark matter with t-channel mediator: a simple step beyond contact interaction, Phys. Rev. D89 (2014) 115014, http://dx.doi.org/10.1103/PhysRevD.89.115014, arXiv:1308.0592.

[24] Y. Bai, J. Berger, Fermion portal dark matter, J. High Energy Phys. 1311 (2013) 171, http://dx.doi.org/10.1007/JHEP11(2013)171, arXiv:1308.0612.

[25] A. DiFranzo, K.I. Nagao, A. Rajaraman, T.M.P. Tait, Simplified models for dark matter interacting with quarks, J. High Energy Phys. 1311 (2013) 014, http://dx.doi.org/10.1007/JHEP11(2013)014, arXiv:1308.2679.

[26] M. Beltran, D. Hooper, E.W. Kolb, Z.A. Krusberg, T.M. Tait, Maverick dark matter at colliders, J. High Energy Phys. 1009 (2010) 037, http://dx.doi. org/10.1007/JHEP09(2010)037, arXiv:1002.4137.

[27] J. Goodman, M. Ibe, A. Rajaraman, W. Shepherd, T.M. Tait, et al., Constraints on light majorana dark matter from colliders, Phys. Lett. B695 (2011) 185-188, http://dx.doi.org/10.1016/j.physletb.2010.11.009, arXiv: 1005.1286.

[28] J. Goodman, M. Ibe, A. Rajaraman, W. Shepherd, T.M. Tait, et al., Constraints on dark matter from colliders, Phys. Rev. D82 (2010) 116010, http://dx.doi. org/10.1103/PhysRevD.82.116010, arXiv:1008.1783.

[29] A. Rajaraman, W. Shepherd, T.M. Tait, A.M. Wijangco, LHC bounds on interactions of dark matter, Phys. Rev. D84 (2011) 095013, http://dx.doi. org/10.1103/PhysRevD.84.095013, arXiv:1108.1196.

[30] G. D’Ambrosio, G.F. Giudice, G. Isidori, A. Strumia, Minimal flavor violation: An effective field theory approach, Nuclear Phys. B645 (2002) 155-187, http://dx.doi.org/10.1016/S0550-3213(02)00836-2, arXiv:hep-ph/0207036.

[31] M. Chala, F. Kahlhoefer, M. McCullough, G. Nardini, K. Schmidt-Hoberg Constraining dark sectors with monojets and dijets, J. High Energy Phys. 07 (2015) 089, http://dx.doi.org/10.1007/JHEP07(2015)089, arXiv:1503.05916.

[32] T. Jacques, K. Nordstroem, Mapping monojet constraints onto simplified dark matter models, J. High Energy Phys. 06 (2015) 142, http://dx.doi.org 10.1007/JHEP06(2015)142, arXiv:1502.05721.

[33] G. Belanger, F. Boudjema, A. Pukhov, A. Semenov, MicrOMEGAs4.1: two dark matter candidates, Comput. Phys. Comm. 192 (2015) 322-329, http: //dx.doi.org/10.1016/j.cpc.2015.03.003, arXiv:1407.6129.

[34] M. Backovic, A. Martini, O. Mattelaer, K. Kong, G. Mohlabeng, Direct detection of dark matter with MadDM v.2.0, Phys. Dark Univ. 9-10 (2015) 37-50, http://dx.doi.org/10.1016/j.dark.2015.09.001, arXiv:1505.04190.

[35] Relic density calculation, courtesy T. du Pree, http://cern.ch/LPCC/index. php? page $=$ dm_wg_docs.

[36] G. Hinshaw, et al., WMAP Collaboration Collaboration, Nine-year wilkinson microwave anisotropy probe (WMAP) observations: cosmological parameter results, Astrophys. J. Suppl. 208 (2013) 19, http://dx.doi.org/10.1088/ 0067-0049/208/2/19, arXiv:1212.5226.

[37] P.A.R. Ade, et al., Planck Collaboration Collaboration, Planck 2015 results. XIII. Cosmological parameters, Astron. Astrophys. 594 (2016) A13, http: //dx.doi.org/10.1051/0004-6361/201525830, arXiv:1502.01589.

[38] F. Kahlhoefer, K. Schmidt-Hoberg, T. Schwetz, S. Vogl, Implications of unitarity and gauge invariance for simplified dark matter models, J. High Energy Phys. 02 (2016) 016, http://dx.doi.org/10.1007/JHEP02(2016)016, arXiv: 1510.02110

[39] G. Gelmini, P. Gondolo, DM production mechanisms, arXiv:1009.3690, 2010.

[40] D.S. Akerib, et al., Improved limits on scattering of weakly interacting massive particles from reanalysis of 2013 LUX data, Phys. Rev. Lett. 116 (16) (2016) 161301, http://dx.doi.org/10.1103/PhysRevLett.116.161301, arXiv: 1512.03506.

[41] E. Aprile, et al., Physics reach of the XENON1T dark matter experiment, J. Cosmol. Astropart. Phys. 1604 (04) (2016) 027, http://dx.doi.org/10.1088 1475-7516/2016/04/027, arXiv:1512.07501.
[42] R. Agnese, et al., New results from the search for low-mass weakly interacting massive particles with the CDMS low ionization threshold experiment, Phys. Rev. Lett. 116 (7) (2016) 071301, http://dx.doi.org/10. 1103/PhysRevLett.116.071301, arXiv:1509.02448.

[43] G. Angloher, et al., Results on light dark matter particles with a lowthreshold CRESST-II detector, Eur. Phys. J. C76 (2016) 25, http://dx.doi.org/ 10.1140/epjc/s10052-016-3877-3, arXiv:1509.01515.

[44] C. Amole, et al., Improved dark matter search results from PICO-2L Run 2, Phys. Rev. D93 (6) (2016) 061101, http://dx.doi.org/10.1103/PhysRevD.93. 061101, arXiv:1601.03729.

[45] C. Amole, et al., Dark matter search results from the PICO-60 $\mathrm{CF}_{3} \mathrm{i}$ bubble chamber, Phys. Rev. D93 (5) (2016) 052014, http://dx.doi.org/10.1103/ PhysRevD.93.052014, arXiv:1510.07754.

[46] D.S. Akerib, et al., Results on the spin-dependent scattering of weakly interacting massive particles on nucleons from the run 3 data of the LUX experiment, Phys. Rev. Lett. 116 (16) (2016) 161302, http://dx.doi.org/10. 1103/PhysRevLett.116.161302, arXiv:1602.03489.

[47] DMTools, http://dmtools.brown.edu.

[48] M. Hoferichter, J. Ruiz de Elvira, B. Kubis, U.-G. Meißner, High-precision determination of the pion-nucleon $\sigma$ term from roy-steiner equations, Phys. Rev. Lett. 115 (2015) 092301, http://dx.doi.org/10.1103/PhysRevLett. 115.092301, arXiv:1506.04142.

[49] P. Junnarkar, A. Walker-Loud, Scalar strange content of the nucleon from lattice QCD, Phys. Rev. D87 (2013) 114510, http://dx.doi.org/10.1103/ PhysRevD.87.114510, arXiv:1301.1114.

[50] A. Ringwald, L.J. Rosenberg, G. Rybka, Axions and other similar particles in the review of particle physics, Chin.Phys. C38 (2014) 090001, http: //pdg.lbl.gov/2015/reviews/rpp2015-rev-axions.pdf.

[51] J.R. Ellis, K.A. Olive, C. Savage, Hadronic uncertainties in the elastic scattering of supersymmetric dark matter, Phys. Rev. D77 (2008) 065026 , http://dx.doi.org/10.1103/PhysRevD.77.065026, arXiv:0801.3656.

[52] U. Haisch, F. Kahlhoefer, T.M.P. Tait, On mono-w signatures in spin-1 simplified models, Phys. Lett. B760 (2016) 207-213, http://dx.doi.org/10. 1016/j.physletb.2016.06.063, arXiv:1603.01267.

[53] M.G. Aartsen, et al., Improved limits on dark matter annihilation in the sun with the 79-string icecube detector and implications for supersymmetry, J. Cosmol. Astropart. Phys. 1604 (04) (2016) 022, http://dx.doi.org/10.1088/ 1475-7516/2016/04/022, arXiv:1601.00653.

[54] K. Choi, et al., Search for neutrinos from annihilation of captured low-mass dark matter particles in the Sun by Super-Kamiokande, Phys. Rev. Lett. 114 (2015) 141301, http://dx.doi.org/10.1103/PhysRevLett.114.141301, arXiv: 1503.04858 .

[55] J. Silk, K.A. Olive, M. Srednicki, The photino, the sun and high-energy neutrinos, Phys. Rev. Lett. 55 (1985) 257-259, http://dx.doi.org/10.1103/ PhysRevLett.55.257.

[56] J. Heisig, M. Kramer, M. Pellen, C. Wiebusch, Constraints on majorana dark matter from the LHC and icecube, Phys. Rev. D93 (5) (2016) 055029, http://dx.doi.org/10.1103/PhysRevD.93.055029, arXiv:1509.07867.

[57] G. Busoni, A. De Simone, W.-C. Huang, On the minimum dark matter mass testable by neutrinos from the sun, J. Cosmol. Astropart. Phys. 1307 (2013) 010, http://dx.doi.org/10.1088/1475-7516/2013/07/010, arXiv:1305.1817.

[58] M. Ackermann, et al., Searching for dark matter annihilation from milky way dwarf spheroidal galaxies with six years of fermi large area telescope data, Phys. Rev. Lett. 115 (2015) 231301, http://dx.doi.org/10.1103/ PhysRevLett.115.231301, arXiv:1503.02641.

[59] M. Ajello, et al., Fermi-LAT observations of high-energy $\gamma$-ray emission toward the galactic center, Astrophys. J. 819 (1) (2016) 44, http://dx.doi. org/10.3847/0004-637X/819/1/44, arXiv:1511.02938.

[60] M. Cirelli, G. Corcella, A. Hektor, G. Hutsi, M. Kadastik, P. Panci, M. Raidal, F. Sala, A. Strumia, PPPC 4 DM ID: A poor particle physicist cookbook for dark matter indirect detection, J. Cosmol. Astropart. Phys. 1103 (2011) 051, http://dx.doi.org/10.1088/1475-7516/2012/10/E01, http: //dx.doi.org/10.1088/1475-7516/2011/03/051, arXiv:1012.4515; JCAP 1210 (2012) E01, Erratum:

[61] O. Buchmueller, S.A. Malik, C. McCabe, B. Penning, Constraining dark matter interactions with pseudoscalar and scalar mediators using collider searches for multijets plus missing transverse energy, Phys. Rev. Lett. 115 (2015) 181802, http://dx.doi.org/10.1103/PhysRevLett.115.181802, arXiv: 1505.07826 .

[62] G. Steigman, B. Dasgupta, J.F. Beacom, Precise relic WIMP abundance and its impact on searches for dark matter annihilation, Phys. Rev. D86 (2012) 023506, http://dx.doi.org/10.1103/PhysRevD.86.023506, arXiv:1204.3622. 